- 研究报告・

\title{
盗蜜对角蒿传粉者行为和生殖成功的影响
}

\author{
杜家潚 1,2 孟 璐 ${ }^{1}$ 孙海芹 ${ }^{2 *}$ 包 颖 ${ }^{*}$ \\ 1 (曲阜师范大学生命科学学院, 山东曲阜 273165) \\ 2 (中国科学院植物研究所系统与进化植物学国家重点实验室, 北京 100093)
}

\begin{abstract}
摘要: 一些研究显示盗蜜对自交植物的结实和结䊏没有显著影响。然而, 对于既有传粉者为其传粉实现异交又能 通过自交实现生殖保障的兼性自交植物来说, 盗蜜对其生殖的影响还知之甚少。由于兼性自交植物可以自交, 盗 蜜对其总体结实可能不会有显著影响, 但可能会通过影响传粉者行为而影响传粉者介导的结实。为了验证这一假 说, 本研究以兼性自交的一年生角蒿(Invarvillea sinensis var. sinensis)为研究材料, 通过野外调查和控制实验, 探 讨了盗蜜对传粉者介导的结实(传粉者行为)和总体结实率的影响。结果表明: 角蒿的盗蜜者和主要传粉者相同, 均 为密林熊蜂(Bombus patagiatus)。熊蜂盗蜜频率平均为 $20.24 \%$ (范围为 $0-51.43 \%$ )。盗蜜对角蒿总体结实率、每果 结籽数和每果种子重量没有显著影响。然而, 被盗蜜花的柱头闭合比率显著高于未被盗蜜花, 说明盗蜜影响传粉 者的访花行为和传粉者介导的结实率。另外, 被盗蜜花的高度显著高于未被盗蜜花, 说明盗蜜者倾向于从较大较 高的花上盗蜜。这些结果为全面认识盗蜜对植物生殖的影响提供了新的信息。
\end{abstract}

关键词: 角蒿属, 兼性自交, 花高度, 盗蜜, 传粉者介导的结实, 生殖

\section{Effects of nectar robbing on pollinator behavior and pollination success in facultative selfing Incarvillea sinensis var. sinensis}

\author{
Jiaxiao $\mathrm{Du}^{1,2}$, Lu Meng ${ }^{1}$, Haiqin Sun ${ }^{2 *}$, Ying Bao ${ }^{1^{*}}$ \\ 1 College of Life Sciences, Qufu Normal University, Qufu, Shandong 273165 \\ 2 State Key Laboratory of Systematic and Evolutionary Botany, Institute of Botany, Chinese Academy of Sciences, Beijing \\ 100093
}

\begin{abstract}
Some plants benefit from self-fertilization for reproductive assurance, and thus might experience little effect of nectar robbing on fruit production. In facultative selfing plants which involve both outcrossing by pollinators and selfing when pollinators are scare or not available, nectar robbing is expected to have no influence on fruit set, but may affect pollinator-mediated fruit set. In order to test this prediction, we manipulated robbing (open to be robbed or excluding nectar robbers by caging flowers) in an annual facultative selfing plant Invarvillea sinensis var. sinensis and quantified fruit set, seed number and weight per fruit. Pollinator-mediated fruit set was evaluated on the basis of the closure of stigmatic lobes, which generally occurs after pollinator visiting. The height of flowers was measured to test whether nectar robbers have a visiting preference for specific floral traits. Bumble bees, workers of Bombus patagiatus, were the primary pollinators of $I$. sinensis and some of them were also nectar robbers. Robbers stole nectar from both open flowers and flower buds. Averaged frequency of nectar robbing was $20.24 \%$, ranging from 0 to $51.43 \%$. Experiments excluding nectar robbers showed that nectar robbing did not have significant effects on proportion of fruit set, seed number per fruit and seed mass per fruit. However, the proportion of stigmatic lobes closed was significantly higher in robbed flowers than in unrobbed flowers, suggesting that robbing influences pollinator-mediated fruit set. Height of robbed flowers was significantly higher than that of unrobbed flowers, suggesting that nectar robbers prefer robbing from larger and higher flowers. These results provide insight into the effects of nectar robbing on the reproduction of plants.
\end{abstract}

收稿日期: 2015-04-27; 接受日期: 2015-06-23

基金项目: 国家自然科学基金(31370403)

* 共同通讯作者 Coauthors for correspondence. E-mail: hqsun@ibcas.ac.cn; baoyingus@126.com 
Key words: Invarvillea, facultative selfing, flower height, nectar robbing, pollinator-mediated fruit set, reproduction

在动植物之间的互作关系中, 盗蜜(nectar robbing)是一种不同于一般传粉者的非正常访花行为 (张彦文等, 2006)。盗蜜特指昆虫、鸟类或其他访花 者在花冠筒上直接打洞从中取食花蜜、但不从花冠 口进入花内给花受粉的现象(Inouye, 1980; Maloof \& Inouye, 2000)。在有花植物中, 盗蜜现象非常普 遍, 但被盗蜜植物主要是具长花管或花上有蜜腺距 的产蜜植物。Irwin和Maloof (2002)统计认为有盗蜜 现象存在的植物有59科214种。到目前为止, 在越来 越多的植物中发现盗蜜现象。常见的盗蜜者是蜂类, 尤以熊蜂(Bombus)和木蜂 (Xylocopa) 为多, 在美洲 一些鸟类也是常见的盗蜜者 (Maloof \& Inouye, 2000; 张彦文等, 2006)。

盗蜜对植物的影响有两个方面, 一个是直接影 响, 即盗蜜会直接导致植物生殖器官柱头和花药的 损坏(Irwin et al., 2010); 另外一个是间接影响, 即 通过影响传粉者的行为来影响植物的适合度(Irwin et al., 2010; Zhang et al., 2014)。自达尔文时代, 盗蜜 被认为对被访植物有不利影响, 但是过去 50 年的研 究显示, 盗蜜还有积极或中性影响。一些研究显示, 通过影响传粉者行为, 盗蜜者会增加花粉的散布距 离、促进异交(Maloof \& Inouye, 2000; Irwin et al., 2010)。例如, Irwin (2003)对假番薯属植物鲜红吉利 草(Ipomopsis aggregata)的研究显示, 盗蜜显著减少 了同株异花受粉, 低水平盗蜜的植物花接受同株花 粉是高水平盗蜜植物花的2倍多。

盗蜜者对植物的影响表现出的复杂性, 一方面 取决于盗蜜者的身份、传粉者的身份和盗蜜者盗取 的花蜜量(Maloof \& Inouye, 2000), 另外还可能与植 物的交配系统有关。例如, Zhang等(2009)对3种紫堇 属(Corydalis)植物的研究显示, 盗蜜对异交种神农 架紫堇(C. ternatifolia)的结籽率有显著负影响, 对 兼性异交的刻叶紫堇(C. incisa)有正影响, 而对自 交的毛黄革(C. tomentella)则无影响。对于兼性自交 植物, 当传粉者稀少、存在不能传粉结实的可能时, 植物会采取自交来保证繁殖。由此可以推测, 盗蜜 对兼性自交植物的结实率可能不会有显著影响, 但 可能会影响传粉者行为, 从而影响传粉者介导的结
实。然而, 到目前为止, 很少有研究来探讨这种可 能性。兼性自交植物广泛存在, 尤其是在有环境胁迫 的条件下, 约 $40 \%$ 的有花植物采取这种生殖方式 (Goodwillie et al., 2005)。对具有混交系统尤其是兼性 自交的植物, 从传粉者介导的结实角度了解盗蜜的影 响, 可以更全面地认识盗蜜对植物适合度的影响。

一些研究显示, 传粉者对花的表型如花大小、 数目等有选择偏好(Harder \& Johnson, 2009)。盗蜜 者作为访花昆虫, 是否对植物表型有访问偏好? 为 什么有的花容易被盗蜜, 而有的则不然? 到目前为 止, 这方面的研究实例相对较少(Navarro \& Medel, 2009)。Traveset等(1998)对倒挂金钟(Fuchsia magellanica)的研究发现, 盗蜜鸟(Phrygilus patagonicus)对浅色花的盗蜜率显著低于对正常色花(红色)。 相对于短花冠管的花, 鸟类更喜欢从长花冠管的花 上盗蜜(Lara \& Ornelas, 2001)。了解盗蜜者对花表型 的访问偏好可以增加我们对选择媒介的认识。

角高(Incarvillea sinensis var. sinensis)是一年生 兼性自交植物, 毛乌素沙地角高种群的自交率较 高, 平均为0.7 (范围为0.6-0.8) ( $\mathrm{Yu}, 2010) 。 \mathrm{Qu}$ 等 (2007)通过去雄实验发现, 角蒿的结实率为 $85.93 \%$, 而传粉者对角蒿总体结实率的贡献为 $56.9 \%$, 自花 受粉对总体结实的贡献为剩余的 $43.1 \%$ 。李晓杰 (2008) ${ }^{\circledR}$ 在其硕士论文中提到角蒿有盗蜜现象, 但 并未对其作进一步研究。盗蜜是否会影响角蒿传粉 者介导的结实并进一步影响其对总体结实的贡献 尚不可知。因此本研究利用野外调查和控制实验探 讨盗蜜对角蒿生殖成功的影响。拟回答如下问题: (1)角蒿的传粉者和盗蜜者分别是什么? (2)盗蜜者 的盗蜜频率怎样? (3)盗蜜是否对植物总体结实率 和传粉者介导的结实产生影响? (4)盗蜜者是否对 植物表型有访问偏好?

\section{1 材料和方法}

\section{1 研究材料和研究地点}

角蒿是紫葳科角蒿属(Incarvillea)的一年生草

(1) 李晓杰 (2008) 毛乌素沙地两种蜜源植物羊柴和角蒿的传粉生物学 研究). 中国科学院植物研究所硕士学位论文, 北京. 
本植物, 主要分布在中国北方(东北和西北)、西南以 及远东俄罗斯地区海拔500-2,500 $\mathrm{m}$ 的山坡和田间 (Zhang \& Santisuk, 1998; Chen et al., 2012)。尽管在 13-120 cm高的植株上通常长有含1-58朵(平均值 标准误, $7.39 \pm 6.71, N=200$ )粉色花的总状花序, 但多数个体每天只开 1 朵花。单花开放时间很短, 为 5-9 h。角蒿的花冠大且鲜艳, 呈钟状漏斗形, 基部 收缩成细筒, 产生花蜜, 明显适应于长喙昆虫传 粉。然而, 近期研究发现, 毛乌素沙地角蒿种群的 自交率较高, 平均为 0.7 (Yu, 2010)。另外, 每朵花 有 $18,816.27 \pm 326.70$ 粒花粉和 $180.80 \pm 5.30$ 枚胚珠 $(N$ = 30朵花), $\mathrm{P} / \mathrm{O}$ 值为 104.7 (Qu et al., 2007), 属于兼 性自交类型的交配系统(Goodwillie et al., 2005)。角 蒿雌雄同株, 二强雄荵, 冠生, 两对花丝弯曲相向 将花药并排排列在花柱两侧, 花药紧贴花柱, 两 瓣触敏型柱头, 位于花药上方。自交发生在花开放 后期, 仍然张开且未棠蒸的花冠被风吹落, 拖动 冠生雄荵沿花柱向前滑动, 使得花药穿过张开的 柱头裂片, 从而实现延迟自交(Qu et al., 2007)。毛 乌素沙地群体的角蒿花期始于 6 月中下旬, 止于 8 月底。

本研究是2014年6-8月在位于内蒙古鄂尔多斯 草地生态系统国家野外观测研究站 $\left(39^{\circ} 29^{\prime} 37.6^{\prime \prime}\right.$ $\mathrm{N}, 110^{\circ} 11^{\prime} 29.4^{\prime \prime} \mathrm{E}$, 海拔1,300 m)开展的。毛乌素是 我国沙漠化研究的关键地区之一, 位于鄂尔多斯高 原的中部和南部, 属典型的大陆性半干旱气候, 平 均降雨量260-450 mm, 且降水多集中在7-8月, 蒸 发强烈(张新时, 1994)。群落生境为流动、固定和半 固定沙丘, 油蒿(Artemisia ordosica)、羊柴(Hedysarum laeve)、沙鞭(Psammochloa villosa)和角蒿等灌 木及草本为优势种, 且呈斑块状分布。

\section{2 传粉者和盗蜜者的种类确定和盗蜜频率的调查}

Qu (2007)和 Li (2008)对鄂尔多斯群体角蒿的 传粉者种类及其访花行为进行了较为详细的观察。 为了进一步了解盗蜜者与传粉者的种类是否相同, 我们在盛花期对角蒿的访花昆虫和盗蜜昆虫进行 了观察和采集, 用乙酸乙酯杀死后请中国科学院动 物研究所的昆虫专家进行种类鉴定。

为了调查盗蜜者的盗蜜频率, 在盛花期 (7月 19-21日), 随机设置 $1 \mathrm{~m} \times 1 \mathrm{~m}$ 的样方 14 个, 每个样 方有15-43朵花, 样方之间相距至少 $5 \mathrm{~m}$ 以上。观察 期间每天记录样方内花冠基部有盗蜜孔(见下文结
果部分)的花数, 统计盗蜜频率。盛花期根据单位面 积样方内花的数目至少 20 朵来判定。

\section{3 盗蜜对传粉者行为和传粉成功的影响}

为了了解盗蜜对结实结籽的影响, 我们随机选 取 100 株个体, 分成2组, 每组 50 株, 分别作如下 2 种 处理: 一组为排除盗蜜者实验, 用 $5 \mathrm{~mm}$ 孔径的渔网 在开花前罩住, 以排除盗蜜者(连同传粉者)的影响; 另外一组为对照, 不作任何处理, 植物可以接受盗 蜜者和传粉者的访问。果实成熟时统计每株个体的 结果数。用 $t$-检验分析盗蜜对结实率的影响。另外, 随机采集被盗蜜花和未被盗蜜花的果实各 15 个, 带 回实验室, 计数每个果实的结籽数目, 并用高精度 天平称重。用广义线性模型(Normal分布和Identity 关联函数)分析盗蜜对结籽数和种子重量的影响。

曲荣明(2007)和我们的观察均发现, 角蒿的柱 头为触敏性柱头, 传粉者访花完毕后, 原本 2 个张 开的柱头裂片会紧紧闭合, 柱头内表面被完全遮 盖。于海燕(2010) ${ }^{\circledR}$ 的研究显示, 角蒿柱头每天闭合 比率与熊蜂的每天活动情况 (pollinator abundance) 一致。因此, 柱头闭合与否可以作为传粉者访花行 为的指标之一，同时也可以用柱头闭合的比率表示 传粉者介导的结实率。在盛花期调查盗蜜频率的样 方内, 记录每个样方被盗蜜的花数、被盗蜜花柱头 闭合的花数、未被盗蜜的花数以及未被盗蜜花柱头 闭合的花数。由于数据是非正态分布, 我们用独立样 本的非参数检验Mann-Whitney $U$-tests来分析被盗蜜 花和未被盗蜜花的柱头闭合比率来检验盗蜜对传粉 者行为和传粉者介导的结实率是否存在影响。

\section{4 盗蜜者对花高度的偏好选择}

为了检测盗蜜者是否对植物表型性状有选择 偏好, 在观测盗蜜频率的同时, 随机选择被盗蜜花 和未被盗蜜花各110朵, 用卷尺测量花的高度。用广 义线性模型(Normal分布和Identity关联函数)分析盗 蜜者对不同高度的花是否有选择偏好。

\section{2 结果}

\section{1 传粉者和盗蜜者的确定和盗蜜频率}

根据我们2014年的野外观察发现，角蒿的传粉 昆虫有2种, 即密林熊蜂(Bombus patagiatus)工蜂

(1) 于海燕 (2010) 毛乌素沙地角蒿延迟自交的适应性. 中国科学院植 物研究所硕士学位论文, 北京. 
和四条无垫蜂(Amegilla (Amegilla) quadrifasciata (de Villers, 1790))雄蜂。根据观察期间 (从 8:0012:00)访花昆虫的出现次数和访花频率来看, 前者 是主要传粉者。同时, 我们还观察到, 密林熊蜂同 时还是盗蜜者, 盗蜜的方式是沿着花冠管在花冠基 部钻孔吸取花蜜(图1a, b)。密林熊蜂不仅从开放的 花盗取花蜜, 而且还会在待开放的花苞上盗蜜(图1 b)。被盗蜜的花苍会继续开花, 盗蜜留下的孔较小 (图1c, d), 由此推测熊蜂盗蜜可能不会对繁殖器官 造成直接破坏。熊蜂的盗蜜频率为 $0-51.43 \%$ (平均 值土标准误, $20.24 \% \pm 2.98 \%$ )。盗蜜熊蜂盗蜜结束后 偶尔还会正常访问角蒿为其传粉。

\section{2 盗蜜对传粉者行为和传粉成功的影响}

排除盗蜜者(和传粉者)实验显示, 罩网罩的角 蒿结实率比对照低, 但二者并无统计学上的显著差 异 $(t=1.082, P=0.282$ ) (图2a)。类似地, 被盗蜜花每 个果实的种子数和种子重均比未被盗蜜花的高(图 $2 b, c)$, 尽管二者无显著差异 (种子数: $\chi^{2}=1.449, P$ $=0.229$; 种子重: $\left.\chi^{2}=2.357, P=0.125\right)$ 。然而, 被盗
蜜花柱头闭合的比率显著高于未被盗蜜花 $(U=189$, $P=0.04$ )(图3a), 高出 $26.8 \%$, 说明被盗蜜花传粉者 介导的结实率显著高于未被盗蜜花。

\section{3 盗蜜者对植物表型的偏好选择}

盗蜜熊蜂对不同高度的花表现出明显的访问 偏好, 被盗蜜花的高度显著高于未被盗蜜花, 高出 27.32\% ( $\left.\chi^{2}=56.287, P<0.001\right)$ (图3b)。

\section{3 讨论}

相较于传粉者与植物和草食动物与植物之间 的关系, 盗蜜在动植物互作中被认为是一种复杂的 关系, 这不仅因为盗蜜对植物适合度的可能影响是 复杂的, 还在于盗蜜者身份的复杂性。一些植物的 盗蜜者和传粉者不同，盗蜜者只会盗取花蜜而不能 为植物传粉，比如假番薯属植物鲜红吉利草的传粉 者是蜂鸟, 而盗蜜者是熊蜂(Irwin, 2003)。另外一些 植物的盗蜜者尽管不是正常传粉者，但还是能为花 传粉，例如东北延胡索(Corydalis ambigua)的正常 传粉者是蜜蜂(Apis cerana)和黑㓵条蜂(Anthophora
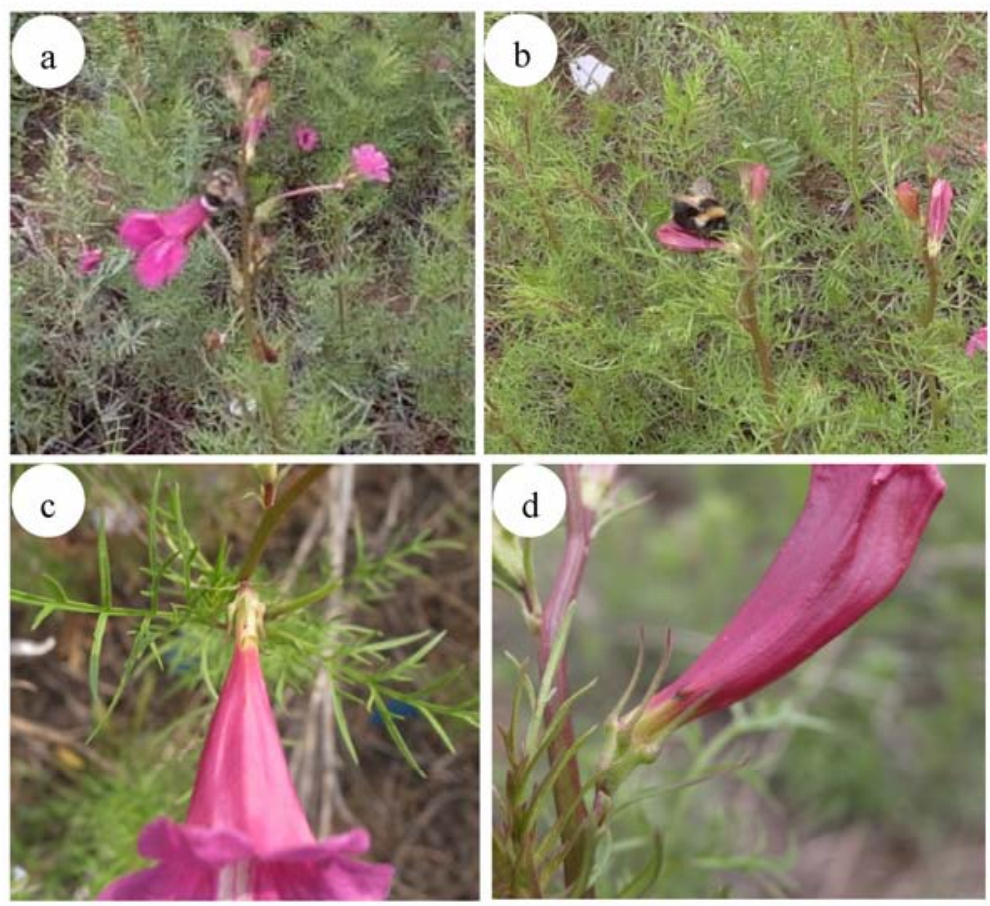

图1 一年生角蒿的盗蜜昆虫和被盗蜜的花和花苞。(a)正在角蒿花上盗蜜的密林熊蜂; (b)正在花苞上盗蜜的密林熊蜂; (c)有 盗蜜孔的角蒿花; (d)有盗蜜孔的花苍。

Fig. 1 Nectar robbers and flowers/buds of Incarvillea sinensis var. sinensis. (a) A bumble bee was robbing nectar from a flower; (b) A bumble bee was robbing nectar from a flower bud; (c) A flower with a hole bitten by a nectar robber; (d) A flower bud with a hole bitten by a nectar robber. 

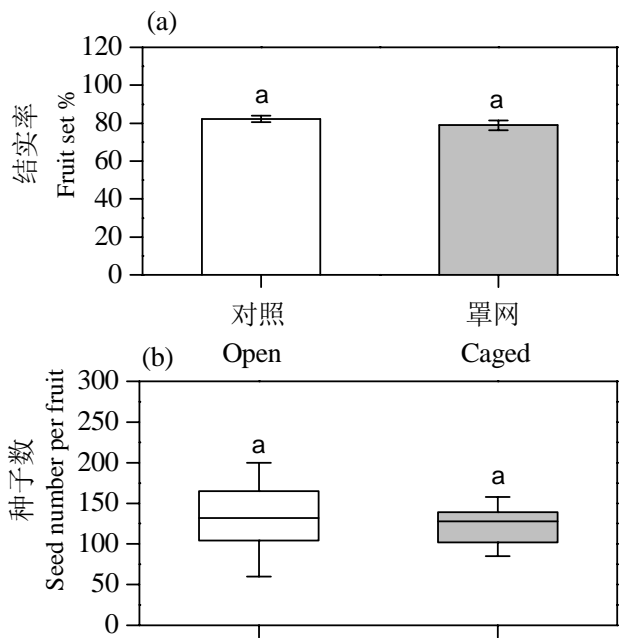

(c)

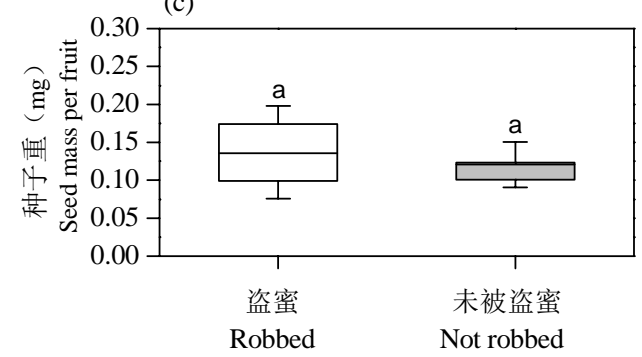

图2 罩网排除角蒿盗蜜者(传粉者)的结实比例(a)以及被盗 蜜花和未被盗蜜花种子数(b)和种子重量(c)。相同字母表示 无显著差异 $(P>0.05)$ 。

Fig. 2 Effects of nectar robbing on fruit set (a), seed number per fruit (b) and seed mass per fruit (c) in Incarvillea sinensis var. sinensis. The same letters indicate no significant differences $(P>0.05)$.

melanognatha), 并且盗蜜熊蜂的传粉效率比正常传 粉者还高(Zhang et al., 2014)。一些盗蜜者之所以能 为植物传粉是因为在盗蜜过程中身体接触到植物 的生殖器官(Navarro, 2000)。本研究发现, 密林熊蜂 不仅是角蒿的传粉者，同时一些个体也是盗蜜者， 这与很多其他植物的盗蜜现象明显不同。角蒿的盗 蜜者无论是从盛开的花上还是花苍上盗取花蜜时, 身体均不会接触到柱头衰退比未被盗蜜花显著低。 尽管角蒿被盗蜜花的每和花药, 因此不能为其传 粉。盗蜜者熊蜂在很多情况下只是盗蜜, 盗蜜结束 后正常访问角高只是偶然现象, 因此我们认为角蒿 的盗蜜熊蜂不是类盗蜜者的传粉者(robber-like pollinators) (Maloof \& Inouye, 2000; Zhang et al., 2014)。至于为什么传粉者类群中的一些个体变为盗 蜜者, 还有待于进一步研究。
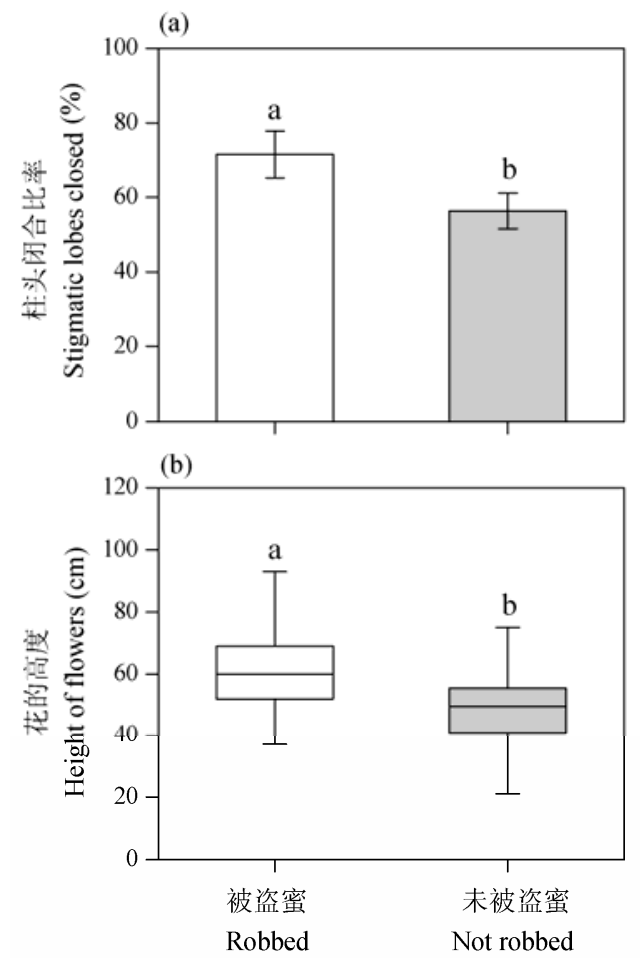

图3 角蒿被盗蜜花和未被盗蜜花柱头闭合比率(a)和花的 高度(b)。不同字母表示显著差异 $(P<0.001)$ 。

Fig. 3 Percentage of stigmatic lobes closed (a) and flower height (b) in robbed flowers and not robbed flowers in Incarvillea sinensis var. sinensis. Different letters indicate significantly different $(P<0.001)$.

角蒿是一种兼性自交植物，排除盗蜜者实验显 示，其结实率并不比对照组的显著低，说明盗蜜对 角蒿的结实在数量上没有影响，即使没有传粉者参 与，角蒿依然能够产生足够多的后代。这一研究结 果与Zhang等(2009)对自交的毛黄堇的研究结果一 致。然而, 盗蜜可能从质量上影响自交植物的适合 度。Zhang等(2011)对青藏高原一年生自交植物喉毛 花(Comastoma pulmonarium)的研究发现，盗蜜对子 代的质量有积极影响，被盗蜜花子代的晚期近交果 结籽数和种子重量与未被盗蜜的相比没有显著差 异, 但盗蜜是否对角蒿子代的质量有影响, 还有待 于下一步研究。

角蒿花大、艳丽，适应于昆虫传粉。曲荣明 (2007) 前期的实验研究发现，去雄花的结实率占角 蒿自然结实率的一半以上(57\%), 说明传粉者传粉 贡献相当大的一部分结实。本研究显示, 尽管盗蜜 对角蒿总体结实率无显著影响, 但是被盗蜜花传粉 
者介导的结实率(柱头闭合比例)却显著高于未被盗 蜜花, 这说明盗蜜可能促进传粉者对花的访问。这 一结果出乎意料。为什么会出现这种结果? 角蒿的 花蜜量较少, 最大花蜜量在花开放后3-4 h, 为 1.41 $\mu \mathrm{L}$, 之后花蜜量迅速下降(Qu et al., 2007; 李晓杰, 2008)。角蒿的单花寿命很短(5-9 h), 我们尚不清楚 盗蜜发生的具体时间是在传粉者访问之前还是之 后。对于盗蜜熊蜂从花苞上盗蜜这一现象, 我们可 以确定盗蜜发生在传粉者访花之前。如果盗蜜发生 在传粉者访花之后, 那么传粉者介导的结实率可能 与盗蜜无关, 是其他因素影响传粉者的访花。但从 盗蜜者的角度考虑, 以快速获取食物为目的而进行 盗蜜的熊蜂为何偏爱从已经被传粉者访问、花蜜非 常少甚至没有花蜜的花上盗蜜? 根据最优受食理 论(optimal foraging theory) (Pyke et al., 1977; Parker \& Smith, 1990), 这种可能性似乎不大。如果盗蜜发 生在花开后、传粉者访花前, 传粉者可能不能识别 已被盗蜜的花(盗蜜孔较小且位于花冠管底部)而继 续访花(Maloof \& Inouye, 2000), 但为何传粉者访问 被盗蜜花的比例会显著高于未被盗蜜花?

盗蜜熊蜂作为访花昆虫对被访问的花表现出 一定的偏好: 被盗蜜的角蒿花的高度显著高于未被 盗蜜的花, 说明盗蜜熊蜂偏爱较高的花。角蒿植株 (花)的高矮与花的大小成正相关关系 (Pearson相关 分析, $r=0.454, \quad P<0.001, N=120)$, 而花的大小 常常与花蜜的多少密切相关(Primack, 1987)。盗蜜 熊蜂偏爱从较高的花上盗蜜, 说明能从更大花上获 取更多的花蜜和能量。一些研究显示, 传粉者也偏 爱访问花大、花多的植物(Harder \& Johnson, 2009)。 我们尚不清楚角高的传粉者是否对大而高的花有 访问偏好, 如果传粉者和盗蜜者具有类似的访问偏 好, 那么这就很好地解释了上面被盗蜜花的传粉者 介导的结实率比未被盗蜜的花高, 同时也说明可能 是花的表型性状(如大小或高矮)而不是盗蜜影响了 传粉者的访问。如果要拆分盗蜜和花表型的影响, 需要进一步开展因素控制实验来验证。

盗蜜在有花植物中是一个普遍现象, 具有重要 的进化和生态意义(Maloof \& Inouye, 2000; 张彦文 等, 2006; Irwin et al., 2010)。盗蜜者对植物的影响是 有利的(mutualists)还是消极的(cheaters), 不仅需要 从数量上来分析, 还需要从质量上(Zhang et al., 2011) 以及用多个适合度组分来探讨。更重要的是,
研究动植物关系时, 不应该忽略盗蜜者可能对植物 生态和进化的影响, 因为作为访花昆虫, 盗蜜者对 植物表型性状可能有选择作用, 可能会与传粉者相 互作用影响植物的进化。然而, 这方面的信息很少。 本研究为理解盗蜜与植物之间的关系提供了有价 值的资料。

致谢: 感谢罗毅波研究员和内蒙古鄂尔多斯草地 生态系统国家野外科学观测研究站(鄂尔多斯市伊 金霍洛旗)对本研究的支持, 感谢中国科学院动物研 究所姚建和袁峰东两位老师对昆虫标本的鉴定。

\section{参考文献}

Chen S, Xing Y, Su T, Zhou Z, Dilcher ED, Soltis D (2012) Phylogeographic analysis reveals significant spatial genetic structure of Incarvillea sinensis as a product of mountain building. BMC Plant Biology, 12, 58.

Goodwillie C, Kalisz S, Eckert CG (2005) The evolutionary enigma of mixed mating systems in plants: occurrence, theoretical explanations, and empirical evidence. Annual Review of Ecology, Evolution and Systematics, 36, 47-79.

Harder LD, Johnson SD (2009) Darwin's beautiful contrivances: evolutionary and functional evidence for floral adaptation. New Phytologist, 183, 530-545.

Inouye DW (1980) The terminology of floral larceny. Ecology, 61, 1251-1253.

Irwin RE (2003) Impact of nectar robbing on estimates of pollen flow: conceptual predictions and empirical outcomes. Ecology, 84, 485-495.

Irwin RE, Bronstein JL, Manson JS, Richardson L (2010) Nectar robbing: ecological and evolutionary perspectives. Annual Review of Ecology, Evolution and Systematics, 41, 271-292.

Irwin RE, Maloof JE (2002) Variation in nectar robbing over time, space, and species. Oecologia, 133, 525-533.

Lara C, Ornelas J (2001) Preferential nectar robbing of flowers with long corollas: experimental studies of two hummingbird species visiting three plant species. Oecologia, 128, 263-273.

Maloof JE, Inouye DW (2000) Are nectar robbers cheaters or mutualists? Ecology, 81, 2651-2661.

Navarro L (2000) Pollination ecology of Anthyllis vulneraria subsp. vulgaris (Fabaceae): nectar robbers as pollinators. American Journal of Botany, 87, 980-985.

Navarro L, Medel R (2009) Relationship between floral tube length and nectar robbing in Duranta erecta L. (Verbenaceae). Biological Journal of the Linnean Society, 96, 392-398.

Parker GA, Smith JM (1990) Optimality theory in evolutionary biology. Nature, 348, 27-33.

Primack RB (1987) Relationships among flowers, fruits, and seeds. Annual Review of Ecology and Systematics, 18, 
409-430.

Pyke GH, Pulliam HR, Charnov EL (1977) Optimal foraging: a selective review of theory and tests. Quarterly Review of Biology, 52, 137-154.

Qu RM (曲荣明) (2007) Pollination Strategy of Plants in Sand Land: Case Studies in Mu Us (沙地中植物的传粉对策—— 毛乌素案例研究). PhD dissertation, Institute of Botany, Chinese Academy of Sciences, Beijing. (in Chinese)

Qu R, Li X, Luo Y, Dong M, Xu H, Chen X, Dafni A (2007) Wind-dragged corolla enhances self-pollination: a new mechanism of delayed self-pollination. Annals of Botany, 100, 1155-1164.

Traveset A, Willson MF, Sabag C (1998) Effect of nectar-robbing birds on fruit set of Fuchsia magellanica in Tierra Del Fuego: a disrupted mutualism. Functional Ecology, 12, 459-464.

Zhang C, Irwin RE, Wang Y, He YP, Yang YP, Duan YW (2011) Selective seed abortion induced by nectar robbing in the selfing plant Comastoma pulmonarium. New Phytologist, 192, 249-255.
Zhang XS (张新时) (1994) Principles and optimal models for development of Maowusu sandy grassland. Journal of Plant Ecology (植物生态学报), 18, 1-16.(in Chinese with English abstract)

Zhang YW (张彦文), Wang Y (王勇), Guo YH (郭友好) (2006) The effects of necar robbing on plant reproduction and evolution. Journal of Plant Ecology (植物生态学报), 30, 695-702. (in Chinese with English abstract).

Zhang YW, Yu Q, Zhao JM, Guo YH (2009) Differential effects of nectar robbing by the same bumble-bee species on three sympatric Corydalis species with varied mating systems. Annals of Botany,104, 33-39.

Zhang YW, Zhao JM, Inouye DW (2014) Nectar thieves influence reproductive fitness by altering behaviour of nectar robbers and legitimate pollinators in Corydalis ambigua (Fumariaceae). Journal of Ecology, 102, 229-237.

Zhang Z, Santisuk T (1998) Bignoniaceae. In: Flora of China (eds Wu ZY, Raven PH, Hong DY), Vol. 18, pp. 213. Science Press, Beijing, and Missouri Botanical Garden Press, St. Louis. 